University of Nebraska - Lincoln

DigitalCommons@University of Nebraska - Lincoln

Papers in the Earth and Atmospheric Sciences

Earth and Atmospheric Sciences, Department

2003

\title{
Estimation of hydraulic conductivity from borehole flowmeter tests considering head losses
}

Vitaly A. Zlotnik

University of Nebraska-Lincoln, vzlotnik1@unl.edu

Brian R. Zurbuchen

University of Nebraska-Lincoln

Follow this and additional works at: https://digitalcommons.unl.edu/geosciencefacpub

Part of the Earth Sciences Commons

Zlotnik, Vitaly A. and Zurbuchen, Brian R., "Estimation of hydraulic conductivity from borehole flowmeter tests considering head losses" (2003). Papers in the Earth and Atmospheric Sciences. 273.

https://digitalcommons.unl.edu/geosciencefacpub/273

This Article is brought to you for free and open access by the Earth and Atmospheric Sciences, Department of at DigitalCommons@University of Nebraska - Lincoln. It has been accepted for inclusion in Papers in the Earth and Atmospheric Sciences by an authorized administrator of DigitalCommons@University of Nebraska - Lincoln. 
This article is a U.S. government work, and is not subject to copyright in the United States.

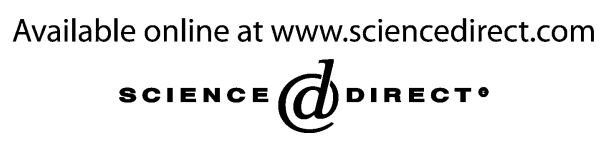

Journal of Hydrology 281 (2003) 115-128

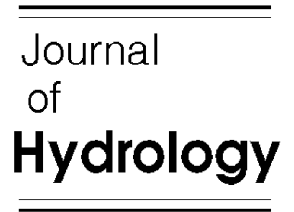

www.elsevier.com/locate/jhydrol

\title{
Estimation of hydraulic conductivity from borehole flowmeter tests considering head losses
}

\author{
Vitaly A. Zlotnik*, Brian R. Zurbuchen ${ }^{1}$ \\ Department of Geosciences, University of Nebraska-Lincoln, Lincoln, NE 68588, USA
}

Received 13 May 2002; accepted 1 May 2003

\begin{abstract}
Recent numerical studies have demonstrated that the conventional interpretation of the borehole flowmeter test (BFT) may lead to considerable errors in estimates of the horizontal hydraulic conductivity $\left(K_{r}\right)$ due to neglect of head loss across the electromagnetic borehole flowmeter (EBF). Even in uniform aquifers, the conventional interpretation underestimates $K_{r}$ at the base and overestimates $K_{r}$ at the top of the aquifer. In this paper, we derive exact analytical solutions for hydraulic head and streamlines induced by the BFT in a confined homogeneous aquifer. The solutions explicitly consider head loss across the EBF. The derived analytical solutions for head distribution in the vicinity of the pumping well and for volumetric flux to the well sections above and below the EBF can be used to interpret field BFT data. In uniform aquifers, this approach can be applied to obtain estimates of $K_{r}$ from the conventional interpretation. Applications of this approach to the BFT field data set from a highly heterogeneous aquifer indicate that the constraint of aquifer homogeneity limits the applicability of this approach, but it can provide useful insights into the mechanism of flux redistribution near the borehole during the BFT.
\end{abstract}

(C) 2003 Elsevier B.V. All rights reserved.

Keywords: Aquifer testing; Hydraulic conductivity; Borehole flowmeter; Head losses; Stream function

\section{Introduction}

The borehole flowmeter test (BFT) methodology to estimate the vertical distribution of horizontal hydraulic conductivity $K_{r}(z)$ in a fully penetrating well is based on measuring the profile of cumulative discharge to a pumped well. There are several flowmeter types that may be applied for this purpose: the heat-pulse flowmeter (Hess, 1986; Paillet et al., 1987), the spinner flowmeter (Hufschmied, 1986;

\footnotetext{
* Corresponding author.

E-mail address: vzlotnik@unl.edu (V.A. Zlotnik).

1 Currently at Nebraska Department of Environmental Quality, 1200 N St, Lincoln, NE 68509, USA.
}

Molz et al., 1989), and the electromagnetic borehole flowmeter (EBF) (Molz and Young, 1993; Young and Pearson, 1995). The EBF has enjoyed more widespread usage (Young, 1995; Boman et al., 1997; Young, 1998) because it is durable, has no moving parts and is sensitive to a wide range of flow rates. However, while the EBF has many advantages, important questions have been raised regarding the device's influence on field measurements (Boman et al., 1997).

To provide a basis for data interpretation, the majority of studies utilize the work of Javandel and Witherspoon (1969) for perfectly stratified aquifers, where $K_{r}$ is a function of the vertical coordinate only. 
Using the assumption of constant head along the well face in an aquifer without ambient flow, they showed that the equipotentials align vertically and become parallel to the well screen surface in the vicinity of the borehole shortly after the pumping start. The drawdown in the more remote parts of the aquifer is still transient. From Darcy's law, the inflow to the well from each distinct layer is proportional to $K_{r}$. To infer $K_{r}$ for each layer, this inflow can be estimated by analysis of the flow rate across the $\operatorname{EBF} Q\left(z_{\mathrm{f}}\right)$, which is the cumulative flow rate that is collected over the well section below the EBF and a function of the EBF coordinate $z_{\mathrm{f}}$ (Fig. 1). Necessarily, the cumulative flow rate in pumping conditions at the top of the aquifer $\left(z_{\mathrm{f}}=b\right)$ is equal to the pump flow rate: $Q_{\mathrm{p}}=Q(b)$.

The resulting method of EBF data interpretation by Molz et al. (1989) is as follows. After measuring flow rate $Q\left(z_{\mathrm{f}, i}\right)$ at different depths $z_{\mathrm{f}, i}, i=1,2, \ldots, n$ and dividing the aquifer into uniform layers of thickness $\Delta z_{\mathrm{f}, i}=z_{\mathrm{f}, i}-z_{\mathrm{f}, i-1}$, the by-layer inflow to the well $Q\left(z_{\mathrm{f}, i}\right)-Q\left(z_{\mathrm{f}, i-1}\right)$ can be found, and the normalized hydraulic conductivity $K_{r, i}$ is calculated from these data

$\frac{K_{r, i}}{\bar{K}_{r}}=\frac{Q\left(z_{\mathrm{f}, i}\right)-Q\left(z_{\mathrm{f}, i-1}\right)}{Q_{\mathrm{p}}} \frac{b}{\Delta z_{\mathrm{f}, i}} \approx \frac{\mathrm{d} Q\left(z_{\mathrm{f}, i}\right)}{\mathrm{d} z} \frac{b}{Q_{\mathrm{p}}}$,

$i=1,2, \ldots, n$,

where $\bar{K}_{r}$ is the arithmetic mean of $K_{r}$ over the well length divided into $n$ layers (Molz et al., 1989):

$\bar{K}_{r}=\frac{1}{n} \sum_{i=1}^{n}\left(K_{r}\right)_{i} \Delta z_{\mathrm{f}, i}$.

It is commonly assumed that the well fully penetrates the aquifer of thickness $b=\sum_{i=1}^{n} \Delta z_{\mathrm{f}, i}$. Rehfeldt et al. (1989) published an analysis of various factors affecting the BFT applications (head losses in the well and screen, skin effects, etc.), but this analysis has not been applied directly to field conditions previously. Also, Boman et al. (1997) explored ambient flow in the aquifer as an additional factor to be considered for data interpretation. However, we

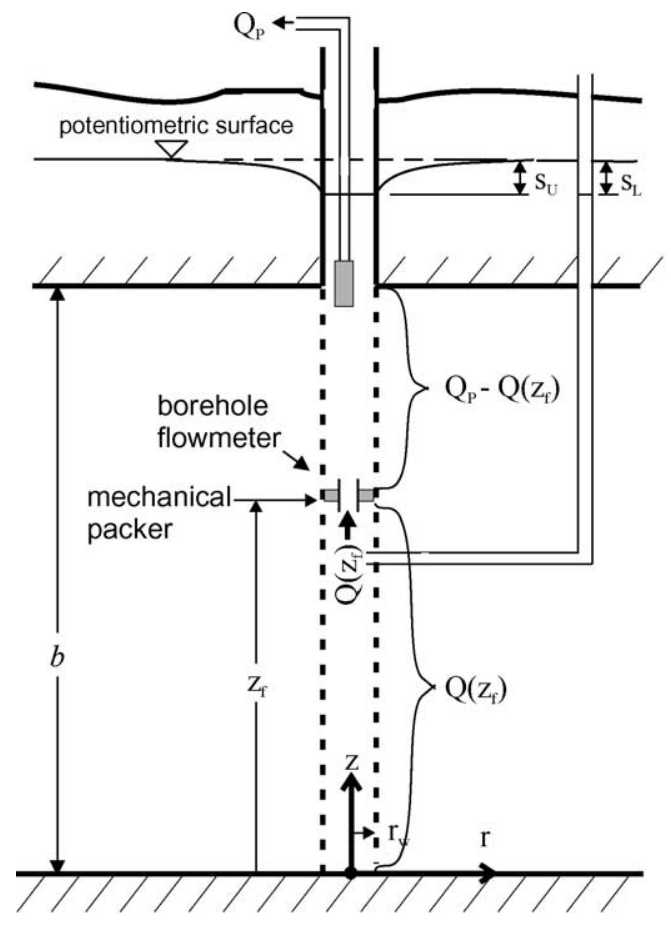

(a)

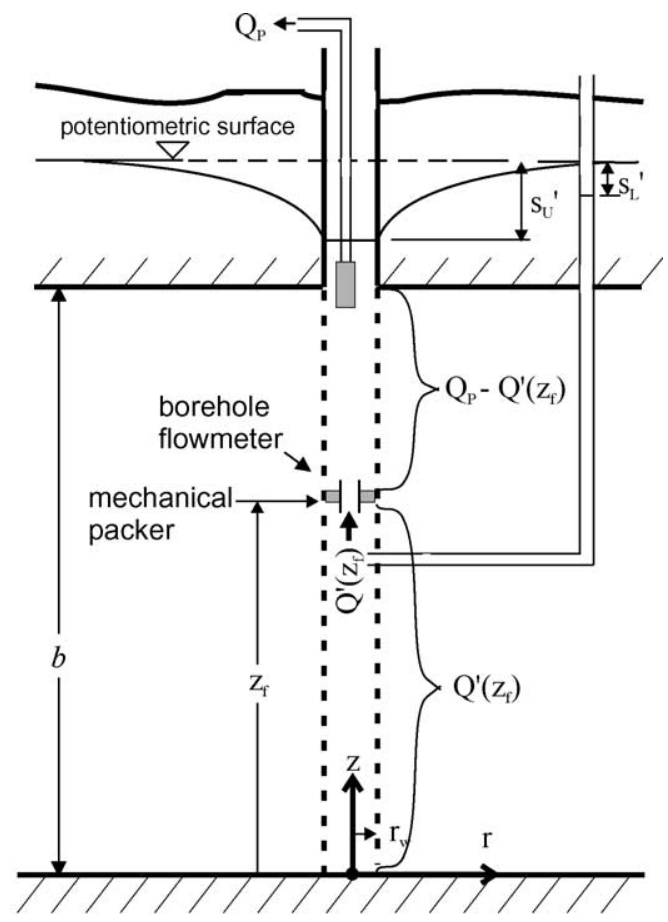

(b)

Fig. 1. Schematic diagram of borehole flowmeter test with electromagnetic borehole flowmeter (EBF): (a) ideal EBF, (b) non-ideal EBF. 
will limit our analysis to aquifers without ambient flow.

Recently, experimental studies of the hydraulic characteristics of the two commercially available EBF devices (flow through orifices of 1.27 and $2.54 \mathrm{~cm}$ ) have found that when operated within their advertised range, significant head losses can occur (Foley, 1997; Arnold, 1997). In highly permeable aquifers, these head losses can be significant relative to the pumping drawdown, which violates the assumption of constant head along the well face. The ramifications of these studies are as follows: (1) the head loss that occurs across the EBF device is a function of flow rate through it; (2) the assumption of constant head along the well face does not hold in the practical ranges of the pumping flow rates; and (3) the well face can be divided into two zones (above and below the EBF location) with different but constant heads. In the case of an ideal EBF without head losses, Eq. (1) is valid. In the case of non-ideal EBF with head losses, this equation needs a correction. Thus, the question is: 'What effect, if any, will an EBF device have on the flow regime surrounding the borehole and the estimates of layer $K_{r}$ ?'

Using numerical simulation of flow near the well with and without a gravel pack, Dinwiddie et al. (1999) found that the conventional interpretation can bias estimates of $K_{r}$ due to the head losses across the EBF. This interpretation underpredicts $K_{r}$ in the lower and intermediate portions of the aquifer and overpredicts $K_{r}$ in the upper portions of the aquifer. The magnitude of this bias is positively correlated with the magnitude of aquifer $K_{r}$ and can skew estimates by more than a factor of two. In cases where a high conductivity gravel pack is present, $K_{r}$ estimates will be further biased.

Ruud et al. (1999) have numerically explored the effects of the head losses, gravel pack, and large-scale aquifer stratification separately and in various combinations. Their results indicated that gravel and aquifer heterogeneity could skew $K_{r}$ values by more than an order of magnitude. Among their practical recommendations, they suggested modification of instruments and/or established field procedures (increase the orifice diameter, use flowmeter without a packer, and perform flowmeter measurements at multiple pumping rates). However, the authors limited their analysis to conceivable 'worst case' scenarios without field examples.

In this paper, we explore an alternative approach-modification of interpretation procedures without changes of field procedures. This interpretation of the BFT takes into account the hydraulic characteristics of the EBF and corrects for head losses. The objectives of this paper are as follows: (1) to derive the head-loss corrected analytical solution of the EBF test problem, and (2) to apply the solution to synthetic and real field data. This solution will be derived for the uniform confined aquifer case, and qualitative aspects of this method will be discussed. We will apply the head-loss corrected interpretation to the cases of a hypothetical uniform aquifer and a real alluvial aquifer. In the latter case, we compare the corrected and uncorrected results with data from multi-level slug tests (MLST). In the homogeneous aquifer, the head-loss corrected interpretation resolves the discrepancy between estimated and actual $K_{r}$, similar to the results of numerical studies by Dinwiddie et al. (1999) and Ruud et al. (1999). However, in highly heterogeneous conditions, the potential of this approach needs further investigation.

\section{Theory}

\subsection{Ideal $E B F$}

Consider a fully penetrating well of radius $r_{\mathrm{w}}$ that is screened throughout the entire thickness $(b)$ of a homogeneous anisotropic confined aquifer of infinite radial extent (Fig. 1). The origin of the system of coordinates is at the base of the well. We will consider the EBF fitted with a short (infinitesimal) packer at elevation $z_{\mathrm{f}}$. Most current data interpretations are based on steady-state BFT data for an ideal EBF, i.e. without head losses. The governing equation of steady-state axisymmetric groundwater flow for a cylindrical coordinate system can be written as follows

$$
\begin{aligned}
& \frac{K_{r}}{r} \frac{\partial}{\partial r}\left(r \frac{\partial s}{\partial r}\right)+K_{z} \frac{\partial^{2} s}{\partial z^{2}}=0, \\
& r>r_{\mathrm{w}}, \quad 0<z<b,
\end{aligned}
$$


where $s$ is drawdown, and $K_{r}$ and $K_{z}$ are the radial and vertical components of hydraulic conductivity. The horizontal confining boundaries are impermeable

$\frac{\partial s(r, 0)}{\partial z}=\frac{\partial s(r, b)}{\partial z}=0, \quad r_{\mathrm{w}} \leq r \leq \infty$

where $r_{\mathrm{w}}$ is the well radius. The boundary condition at the well screen indicates identical drawdowns in the upper well section $\left(s_{\mathrm{U}}\right)$ above and the lower well section $\left(s_{\mathrm{L}}\right)$ below the $\mathrm{EBF}$ position

$s\left(r_{\mathrm{w}}, z\right)=s_{\mathrm{U}}=s_{\mathrm{L}}, \quad 0<z<b$.

At the radius of influence $R$, the drawdown is negligible

$s(R, z)=0, \quad R \rightarrow \infty, \quad 0<z<b$.

The well is being pumped at a constant rate $Q_{\mathrm{p}}$

$Q_{\mathrm{p}}=-2 \pi K_{r} r_{\mathrm{w}} \int_{0}^{b} \frac{\partial s\left(r_{\mathrm{w}}, z\right)}{\partial r} \mathrm{~d} z$

and the drawdown in the aquifer has reached steady-state in the zone of EBF influence $\left(r_{\mathrm{w}}<r<R, 0<z<b\right)$.

The drawdown solution of the problem (2)-(6) obeys the Thiem equation

$s(r, z)=\frac{Q_{\mathrm{p}}}{2 \pi K_{r} b} \ln \left(\frac{R}{r}\right)$,

which yields velocity components $\left(V_{r}, V_{z}\right)$ :

$V_{r}(r, z)=K_{r} \frac{\partial s}{\partial r}=-\frac{Q_{\mathrm{p}}}{2 \pi b r}$,

$V_{z}(r, z)=K_{z} \frac{\partial s}{\partial z}=0$.

Flow rate through the EBF is only a function of its elevation and is as follows:

$Q\left(z_{\mathrm{f}}\right)=-2 \pi K_{r} r_{\mathrm{w}} \int_{0}^{z_{\mathrm{f}}} \frac{\partial s\left(r_{\mathrm{w}}, z\right)}{\partial r} \mathrm{~d} z=Q_{\mathrm{p}} \frac{z_{\mathrm{f}}}{b}$.

Note that velocity components are independent of the radius of influence and that drawdown at the well face $s_{0}$ is

$s\left(r_{\mathrm{w}}, z\right)=\frac{Q_{\mathrm{p}}}{2 \pi K_{r} b} \ln \left(\frac{R}{r_{\mathrm{w}}}\right)=s_{0}=s_{\mathrm{U}}=s_{\mathrm{L}}$.

The flow field near the ideal EBF is shown in Fig. 2.

\subsection{Non-ideal EBF}

In the case when head losses occur across the EBF, the drawdown in the aquifer $s^{\prime}(r, z)$ and the drawdown in well $s^{\prime}\left(r_{\mathrm{w}}, z\right)$ differ from the ideal EBF case. The groundwater flow equation

$\frac{K_{r}}{r} \frac{\partial}{\partial r}\left(r \frac{\partial s^{\prime}}{\partial r}\right)+K_{z} \frac{\partial^{2} s^{\prime}}{\partial z^{2}}=0$,

$r>r_{\mathrm{w}}, \quad 0<z<b$,

boundary conditions on the base and the top of the aquifer

$\frac{\partial s^{\prime}(r, 0)}{\partial z}=\frac{\partial s^{\prime}(r, b)}{\partial z}=0, \quad r_{\mathrm{w}} \leq r \leq \infty$,

and boundary condition at the radius of influence $R$

$s^{\prime}(R, z)=0, \quad R \rightarrow \infty$,

are similar to the case of the ideal EBF. However, the well face is now divided into two sections by the EBF at elevation $z_{\mathrm{f}}$ with a different drawdown in the lower section $\left(s_{\mathrm{L}}^{\prime}\right)$ and the upper well section $\left(s_{\mathrm{U}}^{\prime}\right): s_{\mathrm{U}}^{\prime}>s_{\mathrm{L}}^{\prime}$ :

$s^{\prime}\left(r_{\mathrm{w}}, z\right)=\left\{\begin{array}{ll}s_{\mathrm{U}}^{\prime}, & z_{\mathrm{f}}<z<b \\ s_{\mathrm{L}}^{\prime}, & 0<z<z_{\mathrm{f}}\end{array}\right.$.

To determine $s_{\mathrm{U}}^{\prime}$ and $s_{\mathrm{L}}^{\prime}$ uniquely, two additional conditions are available. The first condition describes the pumping rate

$Q_{\mathrm{p}}=-2 \pi K_{r} r_{\mathrm{w}} \int_{0}^{b} \frac{\partial s^{\prime}\left(r_{\mathrm{w}}, z\right)}{\partial r} \mathrm{~d} z$,

and the other condition

$\Delta s^{\prime}=s_{\mathrm{U}}^{\prime}-s_{\mathrm{L}}^{\prime}=f\left(Q^{\prime}\left(z_{\mathrm{f}}\right)\right)$,

describes the head losses across the EBF, $\Delta s^{\prime}$, as a function of the flow rate through the $\operatorname{EBF} Q^{\prime}\left(z_{\mathrm{f}}\right)$ at elevation $z_{\mathrm{f}}$ :

$Q^{\prime}\left(z_{\mathrm{f}}\right)=-2 \pi K_{r} r_{\mathrm{w}} \int_{0}^{z_{\mathrm{f}}} \frac{\partial s^{\prime}\left(r_{\mathrm{w}}, z\right)}{\partial r} \mathrm{~d} z$ 


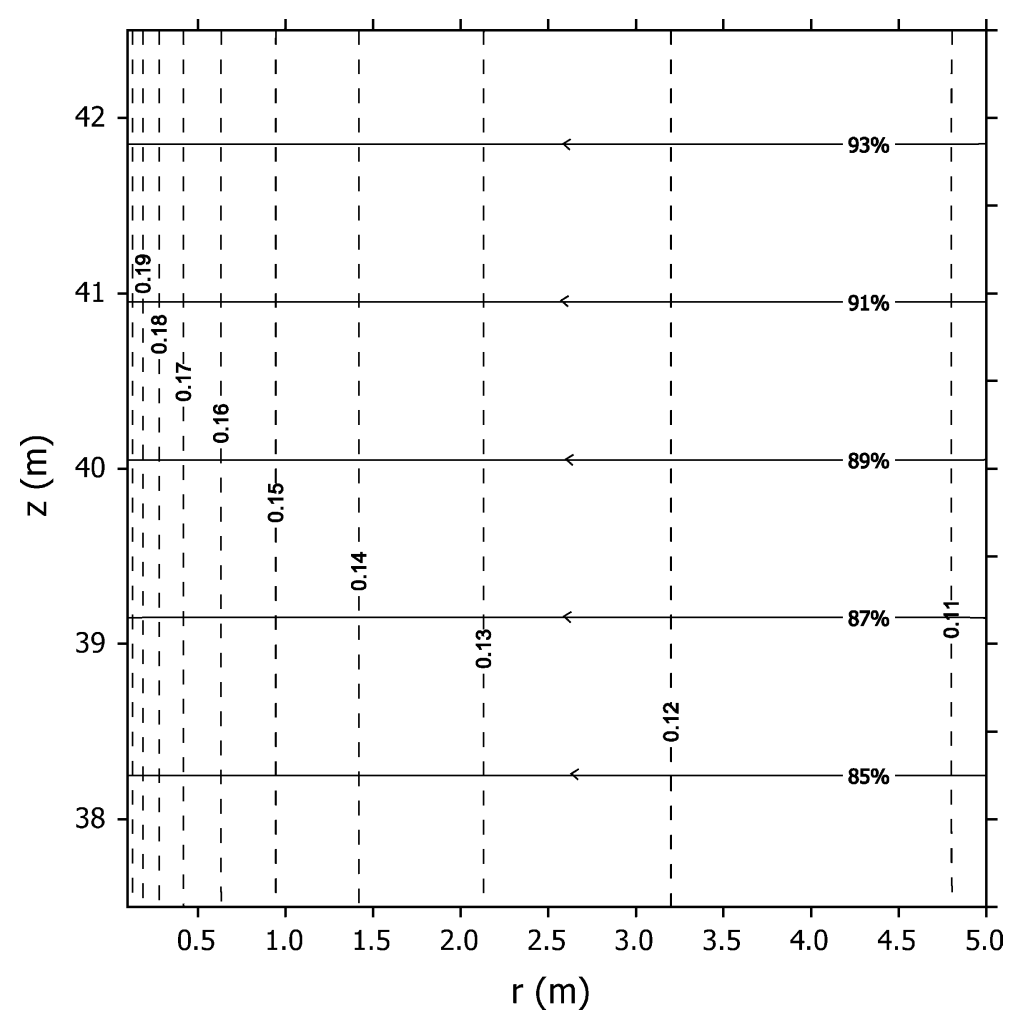

Fig. 2. Groundwater flow near the ideal EBF. Dashed lines show the drawdown, and continuous lines are streamlines that indicate constant values of the normalized Stokes' stream function. Percentile value on each streamline indicates fraction of the total pumping rate that enters the well screen below this streamline.

The non-linearity of condition (16) and the unknown function $Q^{\prime}\left(z_{\mathrm{f}}\right)$ are major obstacles in deriving a solution for the problem defined by Eqs. (11)-(14).

Condition (16) requires additional comment. Parabolic dependence of head losses on flow rate is expected for the nozzle-type configuration of the EBF orifice. Arnold (1997) and Foley (1997) have shown that the head loss for the $1.27 \mathrm{~cm}(1 / 2 \mathrm{in}$.) and $2.54 \mathrm{~cm}$ (1 in.) EBF (Quantum Eng. Corp.) obey the standard head loss equation for a nozzle (Fox and McDonald, 1992)

$\Delta s^{\prime}=s_{\mathrm{U}}^{\prime}-s_{\mathrm{L}}^{\prime}=f\left(Q^{\prime}\left(z_{\mathrm{f}}\right)\right)=C\left(Q^{\prime}\left(z_{\mathrm{f}}\right)\right)^{2}$,

where $C=\left(1-\left(A_{2} / A_{1}\right)^{2}\right) /\left(2 C_{\mathrm{d}}^{2} A_{2}^{2} g\right), A_{1}=\pi r_{\mathrm{w}}^{2}$ is the cross-sectional area of the well screen, $A_{2}=\pi r_{0}^{2}$ is the cross-sectional area of the EBF's orifice, $g=9.81$ $\mathrm{m} / \mathrm{s}^{2}$, and $C_{\mathrm{d}}$ is the empirical discharge coefficient.

Ruud et al. (1999) and Dinwiddie et al. (1999) used the following empirical relationship after Foley
(1997) for the $1.27 \mathrm{~cm}(1 / 2$ in.) EBF:

$$
\begin{aligned}
\Delta s^{\prime} & =s_{\mathrm{U}}^{\prime}-s_{\mathrm{L}}^{\prime}=f\left(Q^{\prime}\left(z_{\mathrm{f}}\right)\right) \\
& =62 Q^{\prime}\left(z_{\mathrm{f}}\right)+4.2 \times 10^{6}\left(Q^{\prime}\left(z_{\mathrm{f}}\right)\right)^{2} .
\end{aligned}
$$

For the $2.54 \mathrm{~cm}$ ( $1 \mathrm{in}$.) $\mathrm{EBF}$, the following equation is valid (Foley, 1997):

$$
\begin{aligned}
\Delta s^{\prime} & =s_{\mathrm{U}}^{\prime}-s_{\mathrm{L}}^{\prime}=f\left(Q^{\prime}\left(z_{\mathrm{f}}\right)\right) \\
& =9.7 Q^{\prime}\left(z_{\mathrm{f}}\right)+2.2 \times 10^{5}\left(Q^{\prime}\left(z_{\mathrm{f}}\right)\right)^{2} .
\end{aligned}
$$

The solution of the boundary value problem (11)(14) with additional conditions (15) and (16) is uniquely defined. This problem is non-linear due to the non-linearity of Eq. (16).

\subsection{Solution of the problem for non-ideal EBF}

Previous studies of the non-ideal EBF by Dinwiddie et al. (1999) and Ruud et al. (1999) utilized 
numerical methods due to the complexity of the problem. However, analysis of the hydraulics indicates that this problem can be reduced to a linear one that lends itself to methods that have been previously utilized by Zlotnik and Ledder (1994, 1996).

We introduce the function $s_{1}(r, z)$ as the difference between the solutions for a non-ideal $\mathrm{EBF}$ and an ideal EBF:

$s^{\prime}(r, z)=s(r, z)+s_{1}(r, z)$.

Substitution of $s^{\prime}$ into the boundary value problem (11)-(17) yields the following boundary value problem for function $s_{1}$

$\frac{K_{r}}{r} \frac{\partial}{\partial r}\left(r \frac{\partial s_{1}}{\partial r}\right)+K_{z} \frac{\partial^{2} s_{1}}{\partial z^{2}}=0$,

$r>r_{\mathrm{w}}, \quad 0<z<b$,

boundary conditions on the base and the top of the aquifer

$\frac{\partial s_{1}(r, 0)}{\partial z}=\frac{\partial s_{1}(r, b)}{\partial z}=0, \quad r_{\mathrm{w}} \leq r \leq \infty$,

and boundary condition at the radius of influence $R$

$s_{1}(R, z)=0, \quad R \rightarrow \infty$,

that are similar to the case of ideal EBF. The drawdown values in the lower section $s_{\mathrm{L}}^{\prime}$ and the upper well section $s_{\mathrm{U}}^{\prime}$ yield the following boundary condition:

$s_{1}\left(r_{\mathrm{w}}, z\right)=\left\{\begin{array}{ll}s_{\mathrm{U}}^{\prime}-s_{0}, & z_{\mathrm{f}}<z<b \\ s_{\mathrm{L}}^{\prime}-s_{0}, & 0<z<z_{\mathrm{f}}\end{array}\right.$.

To determine $s_{\mathrm{U}}^{\prime}$ and $s_{\mathrm{L}}^{\prime}$ uniquely, two additional conditions stemming from Eqs. (15), (16) and (21) are used

$2 \pi K_{r} r_{\mathrm{w}} \int_{0}^{b} \frac{\partial s_{1}\left(r_{\mathrm{w}}, z\right)}{\partial r} \mathrm{~d} z=0$,

$\Delta s^{\prime}=s_{\mathrm{U}}^{\prime}-s_{\mathrm{L}}^{\prime}=f\left(Q^{\prime}\left(z_{\mathrm{f}}\right)\right)$

where the flow rate through the $\mathrm{EBF} Q^{\prime}\left(z_{\mathrm{f}}\right)$ at elevation $z_{\mathrm{f}}$ is defined as follows:

$Q^{\prime}\left(z_{\mathrm{f}}\right)=Q\left(z_{\mathrm{f}}\right)-2 \pi K_{r} r_{\mathrm{w}} \int_{0}^{z_{\mathrm{f}}} \frac{\partial s_{1}\left(r_{\mathrm{w}}, z\right)}{\partial r} \mathrm{~d} z$.
This condition relating readings of the ideal and non-ideal EBF will be used to convert data from one type of EBF to the other.

An important conclusion follows from relationship (26), which is rewritten as follows

$2 \pi K_{r} r_{\mathrm{w}} \int_{0}^{z_{\mathrm{f}}} \frac{\partial s_{1}\left(r_{\mathrm{w}}, z\right)}{\partial r} \mathrm{~d} z=-2 \pi K_{r} r_{\mathrm{w}} \int_{z_{\mathrm{f}}}^{b} \frac{\partial s_{1}\left(r_{\mathrm{w}}, z\right)}{\partial r} \mathrm{~d} z$.

This indicates that the drawdown $s_{1}$ can be attributed to equivalent recirculation well causing a dipole flow field, where the lower section below the flowmeter injects water to the aquifer, while the upper section extracts water from the aquifer with an equivalent flow rate. The kinematic structure of this problem was studied previously in detail by Zlotnik and Ledder (1994, 1996). The non-ideal BFT flow can be described as the superposition of the flow near a vertical dipole (drawdown $s_{1}$ ) and the flow to a vertical sink (drawdown $s$ ).

Neglecting the vertical size of EBF eliminates computational difficulties associated with multiscreen wells (Cole and Zlotnik, 1994; Ruud and Kabala, 1997). The solution of the problem (22)(25) for $R=\infty$ can be written using standard techniques

$s_{1}(r, z)=-\Delta s^{\prime} \sum_{n=1}^{\infty} \frac{b_{n}}{n \pi} \cos \left(\frac{n \pi z}{b}\right) K_{0}\left(\frac{n \pi r}{a b}\right)$,

where

$a=\left(K_{r} / K_{z}\right)^{1 / 2}$,

$b_{n}=2 \sin \left(\frac{n \pi z_{\mathrm{f}}}{b}\right) / K_{0}\left(\frac{n \pi r_{\mathrm{w}}}{a b}\right)$,

and where $K_{0}(x)$ is the modified Bessel's function. The corresponding velocity components are as follows:

$$
\begin{aligned}
V_{r}^{\prime}=K_{r} \frac{\partial s^{\prime}}{\partial r}= & V_{r}+\frac{K_{r} \Delta s^{\prime}}{a b} \sum_{n=1}^{\infty} b_{n} \\
& \times \cos \left(\frac{n \pi z}{b}\right) K_{1}\left(\frac{n \pi r}{a b}\right),
\end{aligned}
$$


$V_{z}^{\prime}=K_{z} \frac{\partial s^{\prime}}{\partial z}=\frac{K_{z} \Delta s^{\prime}}{b} \sum_{n=1}^{\infty} b_{n} \sin \left(\frac{n \pi z}{b}\right) K_{0}\left(\frac{n \pi r}{a b}\right)$

Substitution of Eq. (30) into Eq. (28) and using properties of the modified Bessel's function $\left(K_{0}^{\prime}(x)=-K_{1}(x)\right)$ yield equation for the flow rate across the flowmeter as follows

$Q^{\prime}\left(z_{\mathrm{f}}\right)=Q\left(z_{\mathrm{f}}\right)-\gamma \Delta s^{\prime} F\left(\frac{z_{\mathrm{f}}}{b}, \frac{r_{\mathrm{w}}}{b}, a\right)$,

where $F$ is a shape factor:

$F\left(\frac{z_{\mathrm{f}}}{b}, \frac{r_{\mathrm{w}}}{b}, a\right)=\sum_{n=1}^{\infty} \frac{b_{n}}{n \pi} \sin \left(\frac{n \pi z_{\mathrm{f}}}{b}\right) K_{1}\left(\frac{n \pi r_{\mathrm{w}}}{a b}\right)$,

$\gamma=2 \pi r_{\mathrm{w}}\left(K_{r} K_{z}\right)^{1 / 2}$.

Eq. (34) leads to a solution of the non-linear boundary value problem using the following simple algorithm:

1. For a given EBF elevation $z_{\mathrm{f}}$, the flow rate for an ideal EBF $Q\left(z_{\mathrm{f}}\right)$ is determined from Eq. (9).

2. The shape factor $F$ is evaluated from Eq. (35) that correspond to elevation $z_{\mathrm{f}}$.

3. Eqs. (16) and (34) (or one of more specific equations for head losses (18)-(20)) yield parameters $\Delta s^{\prime}$ and $Q^{\prime}\left(z_{\mathrm{f}}\right)$ that correspond to elevation $z_{\mathrm{f}}$.

4. The distribution of $s_{1}(r, z)$ and $s^{\prime}(r, z)$ is evaluated using Eqs. (21) and (30), respectively.

The presence of a discontinuity in head at the EBF elevation results in a poor convergence of the Fourier series at $r=r_{\mathrm{w}}$ for drawdown and for velocity components. Treatment of these discontinuities was discussed by Hamming (1962). In our case, convergence can be drastically improved if one computes the series in Eqs. (30), (32), (33), and (35) not at the well face $\left(r=r_{\mathrm{w}}\right)$, but at a small distance from the well surface $r=r_{\mathrm{w}}+\varepsilon$, where $\varepsilon \ll r_{\mathrm{w}}$; however, terms $b_{n}$ that are given in Eq. (31) should be computed at $r=r_{\mathrm{w}}$.

\subsection{Stokes' stream function and streamlines}

Ideal EBF. For steady-state axisymmetric flow, the Stokes' stream function $\psi(r, z)$ is defined by the equations (Bear, 1972, p. 229):

$$
\begin{aligned}
& \frac{\partial \psi}{\partial r} \equiv K_{z} r \frac{\partial s}{\partial z}=r V_{z}, \\
& \frac{\partial \psi}{\partial z} \equiv-K_{r} r \frac{\partial s}{\partial r}=-r V_{r} .
\end{aligned}
$$

Contour values of $\psi(r, z)$ represent streamlines, which can be applied to analyze the flow structure near the EBF. The stream function can be defined with arbitrary reference level; for convenience we assign $\psi\left(r_{\mathrm{w}}, 0\right)=0$.

It follows from Eq. (8) and definitions (36) that the Stokes' stream function for flow to the well due to pumping can be written in the following form

$\psi(r, z)=\frac{Q_{\mathrm{p}}}{2 \pi} \frac{z}{b}, \quad 0<z<b$.

Notice that the Stokes' stream function is dependent on relative elevation $(z / b)$ and independent of radial position $r$ of the observation point. The streamlines are parallel to and evenly spaced between the upper and lower boundaries. We introduce the normalized Stokes' stream function

$\Psi(r, z)=\frac{\psi(r, z)}{\psi\left(r_{\mathrm{w}}, b\right)}=\frac{z}{b}, \quad 0<z<b$,

where $\psi(r, z)$ is normalized to its maximum value $\psi\left(r_{\mathrm{w}}, b\right)=Q_{\mathrm{p}} / 2 \pi$ so that $0 \leq \Psi(z) \leq 1$ (Fig. 2).

Non-ideal EBF. We denote Stokes' stream function for non-ideal borehole flowmeter as $\psi^{\prime}(r, z)$ which also satisfies Eq. (36). Substituting Eqs. (32) and (33) into Eq. (36) and using the modified Bessel's function property that was given above, one can integrate Eq. (36) subject to condition $\psi^{\prime}\left(r_{\mathrm{w}}, 0\right)=0$.

Resulting Stokes' stream function $\psi^{\prime}(r, z)$ is a linear combination of two stream functions for flow to the well and for recirculation due to by-pass flow around the EBF and to the head losses across the EBF:

$$
\begin{aligned}
\psi^{\prime}(r, z)= & \psi(r, z)-r\left(K_{r} K_{z}\right)^{1 / 2} \Delta s^{\prime} \sum_{n=1}^{\infty} \frac{b_{n}}{n \pi} \\
& \times \sin \left(\frac{n \pi z}{b}\right) K_{1}\left(\frac{n \pi r}{a b}\right),
\end{aligned}
$$

$r_{\mathrm{w}}<r<\infty, 0<z<b$. 
We introduce the normalized Stokes' stream function

$\Psi^{\prime}(r, z)=\frac{\psi^{\prime}(r, z)}{\psi^{\prime}\left(r_{\mathrm{w}}, b\right)}=\frac{\psi^{\prime}(r, z)}{Q_{\mathrm{p}} / 2 \pi}$,

where $0 \leq \Psi^{\prime}(z) \leq 1$, and the final form can be written as follows

$$
\begin{aligned}
& \Psi^{\prime}(r, z)= \Psi(r, z)-\frac{2 r\left(K_{r} K_{z}\right)^{1 / 2} \Delta s^{\prime}}{Q_{\mathrm{p}}} \sum_{n=1}^{\infty} \frac{b_{n}}{n} \\
& \times \sin \left(\frac{n \pi z}{b}\right) K_{1}\left(\frac{n \pi r}{a b}\right), \\
& r_{\mathrm{w}}<r<\infty, 0<z<b .
\end{aligned}
$$

\section{Applications}

\subsection{Relationship between $K$ estimates from the ideal and non-ideal EBF data}

While field applications of the EBF always have a certain degree of non-ideality due to head losses across the EBF, it is desirable to find the appropriate corrected values of flow rate through the orifice that could be measured by an ideal EBF that does not disturb the horizontal flow in the tested aquifer. The derived equations can be used for finding relations between $K$ estimates from ideal and non-ideal EBF data. We will explore the possibility of correcting data interpretation for homogeneous and heterogeneous aquifers. In all cases we follow the approach by Ruud et al. (1999) and Dinwiddie et al. (1999) who considered only isotropic aquifers:

$K=K_{r}=K_{z}$.

For an ideal EBF, interpretation of field data in a uniform aquifer by Eq. (1) yields the following trivial identity:

$$
\left(\frac{K_{i}}{K}\right)_{\text {ideal }}=1 \text {. }
$$

For a non-ideal EBF in uniform aquifers, substitution of flow rate from Eq. (34) to Eq. (1) yields the following $K$ estimate

$$
\left(\frac{K_{i}}{K}\right)_{\text {non-ideal }}=\left(\frac{K_{i}}{K}\right)_{\text {ideal }}-\delta=1-\delta,
$$

where

$\delta=\frac{2 \pi r_{\mathrm{w}} K b}{Q_{\mathrm{p}}}\left(\frac{\left(\Delta s^{\prime} F\right)_{z=z_{\mathrm{f}, i}}-\left(\Delta s^{\prime} F\right)_{z=z_{\mathrm{f}, i-1}}}{z_{\mathrm{f}, i}-z_{\mathrm{f}, i-1}}\right)$.

Therefore, the final equation for estimates of $K$ due to the non-ideality of EBF can be expressed via the correction $\delta$ :

$\left(\frac{K_{i}}{K}\right)_{\text {ideal }}=\left(\frac{K_{i}}{K}\right)_{\text {non-ideal }}+\delta$.

This equation should be used as follows. In a standard approach, data from non-ideal flowmeter can be processed using Eq. (1) to estimate relative values of $K_{i}$ for each layer. These are $\left(K_{i} / K\right)_{\text {non-ideal }}$ estimates. Then, Eq. (46) is applied to each layer to convert these estimates to $\left(K_{i} / K\right)_{\text {ideal }}$ values that would be obtained using an ideal EBF (without head losses across the flowmeter).

In cases where the EBF has been applied for characterization of relatively uniform and isotropic media similar to one that has been studied by Dinwiddie et al. (1999), this correction could be adequate to remove the non-linearity effects. However, in layered heterogeneous aquifers, the potential of this approach has to be tested.

We will examine the impacts of EBF non-ideality on $K$ estimates using examples by Dinwiddie et al. (1999) and Ruud et al. (1999), and original field data. Previous investigations employed finite-difference models and iterative numerical methods to satisfy mass balance in the borehole. In our case, we will demonstrate that the simple analytical model can yield additional insights into the problem of $K$ measurements.

\subsection{Effect of head losses on large-scale $K$ estimates}

Ruud et al. (1999) investigated several hypothetical cases of EBF applications (uniform aquifer, effect of gravel pack, and combined effect of the gravel pack and two-layer aquifer heterogeneity). They considered a BFT performed in a well of radius $r_{\mathrm{w}}=0.1 \mathrm{~m}$ in uniform isotropic aquifer with $K=$ $5 \times 10^{-5} \mathrm{~cm} / \mathrm{s}$ and saturated thickness $b=45 \mathrm{~m}$. The well is being pumped at a constant rate of $Q_{\mathrm{p}}=20.83$ $1 /$ min. An EBF having a $1 / 2$ in. orifice has the head loss function given by Eq. (19). 
Ruud et al. (1999) evaluated inflow to the well above and below the EBF at only one elevation $z_{\mathrm{f}}=$ $40 \mathrm{~m}$. Multiple runs for different EBF elevations would require consecutive well grid adjustments. Applying Eq. (1), they determined the apparent hydraulic conductivity below the $\operatorname{EBF}\left(K_{\text {below }} / K\right)=$ 0.73 and above the $\operatorname{EBF}\left(K_{\text {above }} / K\right)=3.3$.

Our calculations of the same BFT follow the simple algorithm from Section 2.3. At given EBF elevation $z_{\mathrm{f}, 1}=40$, we calculated the flow rate for an ideal $\operatorname{EBF} Q(40)=3.09 \times 10^{-4} \mathrm{~m}^{3} / \mathrm{s} \quad(18.521 / \mathrm{min})$ from Eq. (9) and $\gamma=3.14 \times 10^{-5} \mathrm{~m}^{2} / \mathrm{s}$. Parameter $F=11.76$ from Eq. (35) was estimated using parameter $\varepsilon=0.005 \mathrm{~m}$. This yields the equation: $Q^{\prime}(40)=3.09 \times 10^{-4}-3.69 \times 10^{-4} \Delta s^{\prime}$ according to Eq. (34), which can be reduced using Eq. (19) to a quadratic equation for $Q^{\prime}(40)$. This yields $Q^{\prime}(40)=$ $2.26 \times 10^{-4} \mathrm{~m}^{3} / \mathrm{s}$ and $\Delta s^{\prime}=0.224 \mathrm{~m}$. Substituting these parameters into the correction $\delta$ with $z_{\mathrm{f}, 0}=0$ and $z_{\mathrm{f}, 2}=45$, we estimated $\left(K_{1} / K\right)=\left(K_{\text {below }} / K\right)=$ 0.73 below the EBF and $\left(K_{2} / K\right)=\left(K_{\text {above }} / K\right)=3.1$ above the EBF. These results correspond well with numerical simulations by Ruud et al. (1999). This algorithm can be easily repeated for any number of elevations.

We can compare flow systems that develop by using an ideal EBF and a non-ideal EBF. The steadystate distribution of drawdown from Eq. (7) and streamlines in the vicinity of the well with an ideal EBF are shown in Fig. 2. The radius of influence was calculated as $R=1.5 \sqrt{K t / S_{\mathrm{s}}}=636 \mathrm{~m}$ using data from Ruud et al. (1999). It is obvious that equipotentials are vertical, streamlines of flow are horizontal, and $K$ estimates above and below the EBF based on Eq. (1) are valid. For the case with head loss across the EBF, the head distribution is calculated from Eqs. (7), (21), and (30) and streamlines obey Eq. (41). Both streamlines and equipotentials are shown in Fig. 3. In this case, streamlines are diverted towards the upper zone, even at large radial distances from the well due to the by-pass flow from the lowest section to the upper section. Flux to the $2.5 \mathrm{~m}$ zone just below the EBF represents only about $5 \%$ of the flux that would be expected for the ideal case. This redistribution of flux through the aquifer has a serious impact on estimates of hydraulic conductivity. Note that this plot refines the streamlines presented by Ruud et al. (1999, their Figures 3 and 4) because our method is based on the exact Stokes' stream function rather than on particle tracking algorithms.

\subsection{Apparent profile of $K$ in uniform aquifers}

Ruud et al. (1999) gave results for one of the worst case scenarios of disparity between the upper and the lower well sections obtained due to head losses in non-ideal EBF. It was demonstrated that streamlines of flow in the aquifer are horizontal when no losses occur across the EBF. However, the head difference between the sections above and below the EBF is a function of flow rate through the $\mathrm{EBF}$, which is in turn a function of the elevation of the EBF. The overall impact of head loss through the EBF results in an apparent profile of $K$ in an aquifer divided into multiple aquifer layers. Dinwiddie et al. (1999) performed these labor intensive computations for multi-layer aquifer. Our method can handle this problem using less effort and with identical accuracy.

To investigate the role of EBF head loss on the $K_{i}$ profile, we consider the data set from Dinwiddie et al. (1999). The BFT was performed in a well of radius $r_{\mathrm{w}}=0.05 \mathrm{~m}$ in an aquifer that has saturated thickness $b=6.1 \mathrm{~m}$. The confined aquifer is isotropic and homogeneous with $K=9.1 \mathrm{~m} /$ day. The well is being pumped at a constant rate of $Q_{\mathrm{p}}=19.99 \mathrm{l} / \mathrm{min}$. We divide the aquifer into 20 layers with a thickness of $\Delta z_{\mathrm{f}}=0.3 \mathrm{~m}$ each, with the exception of the central layer that has a thickness of $0.4 \mathrm{~m}$ (to be fully compatible with work of Dinwiddie et al. (1999)). In this hypothetical scenario, an EBF with a $1 / 2$ in. orifice and $C_{\mathrm{d}}=0.85$ in Eq. (18) is used.

We can theoretically calculate the cumulative discharge $Q^{\prime}\left(z_{\mathrm{f}, i}\right)$ at the boundary of each layer by applying simple algorithm that has been described in Section 2.3 and illustrated in Section 3.2. The obtained profile of $\left(K_{i} / K\right)_{\text {non-ideal }}$ is compared in Fig. 4 with the numerical results of Dinwiddie et al. (1999). The analytical and numerical profiles compare well considering that the numerical experiments assumed a finite packer length of $12.7 \mathrm{~cm}$ and the analytical solution was derived assuming an infinitesimal packer length. Discretization errors in the numerical model may also be accountable for minor discrepancies.

This profile was obtained using the standard EBF interpretation from the cumulative discharge profile 


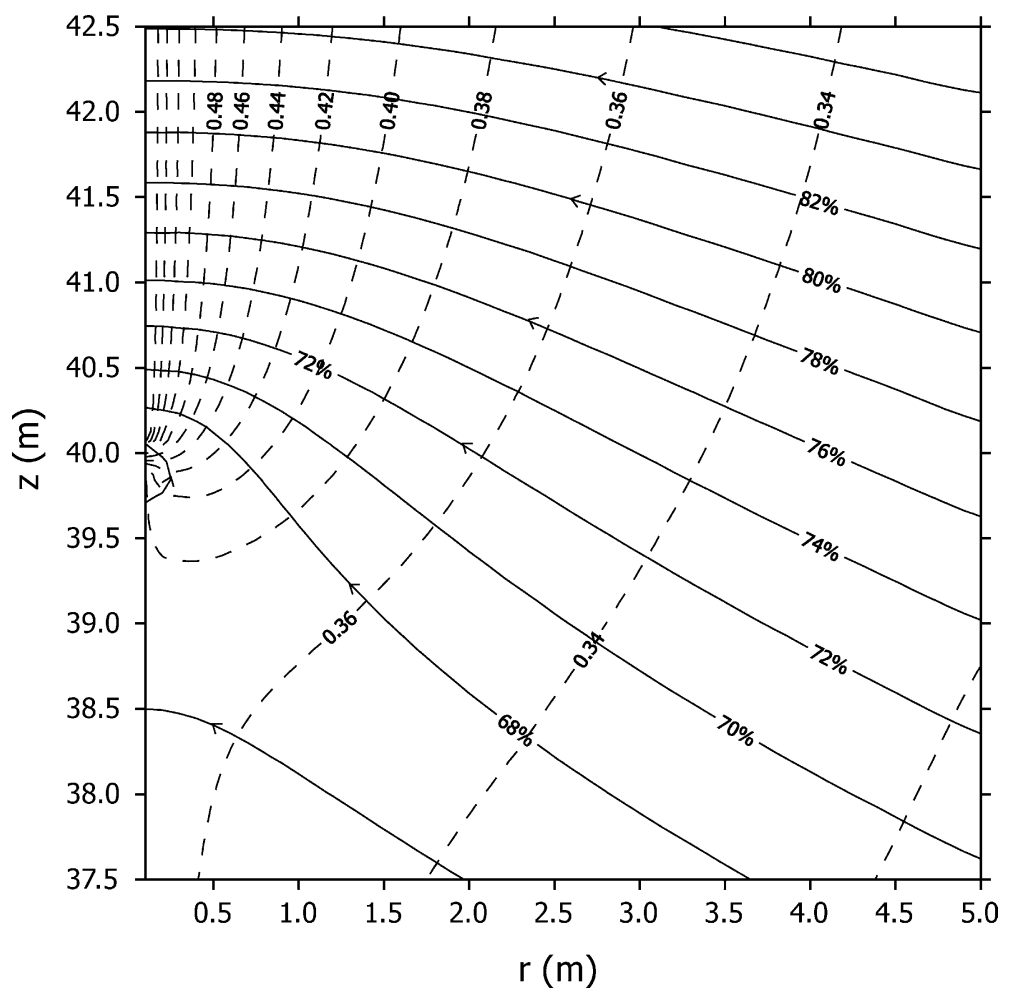

Fig. 3. Groundwater flow near the non-ideal EBF. Dashed lines show the drawdown, and continuous lines are streamlines that indicate constant values of the normalized Stokes' stream function. Percentile value on each streamline indicates fraction of the total pumping rate that enters the well screen below this streamline.

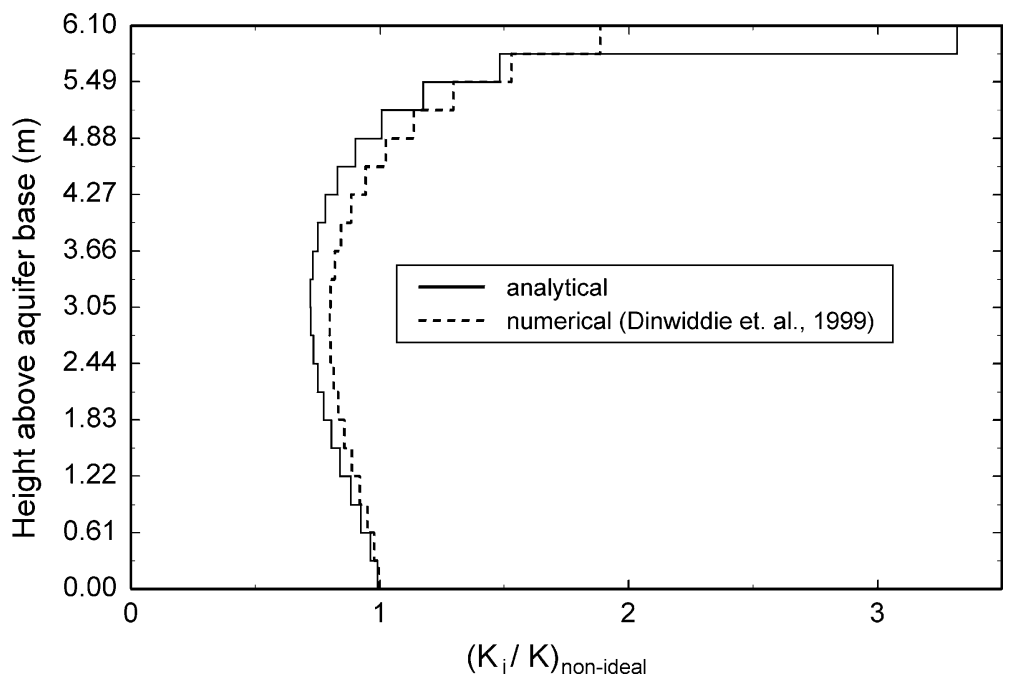

Fig. 4. Comparison of the analytical estimates (this paper) and numerical estimates (Dinwiddie et al., 1999, Table 2) of the apparent hydraulic conductivity obtained from the BFT with non-ideal EBF. 
that considers head losses across the flowmeter. The profile differs significantly from the true aquifer $K$. In the lower $85 \%$ of the aquifer thickness, the $K$ estimate is less than the true aquifer $K$ due to redistribution of flow to the zone above the EBF. The discrepancy is largest near the center of the aquifer. This is intuitive, since as the EBF is positioned higher in the aquifer, head loss across it becomes greater. In the upper sections of the aquifer a greater portion of flux is collected, which results in overestimates of $K$ for this zone.

The apparent results shown in Fig. 4 can be completely corrected for uniform aquifer conditions by using Eq. (46) for each layer. This method can be formally extended to 'relatively uniform' aquifers, because the latter equation requires estimates of average $K$ and shape factor $F$ only. However, validity of this technique for heterogeneous aquifers is not apparent. We will evaluate potential of this technique in heterogeneous aquifers in Section 3.4.

\subsection{Evaluation of correcting field data using an approach for uniform aquifers}

An EBF with a 1 in. flow-through orifice was applied to collect profiles of flow rate in two wells (well 14 and well 15). These wells with $r_{\mathrm{w}}=5.1 \mathrm{~cm}$ fully penetrate the shallow unconfined aquifer at the Management Systems Evaluation area (Zlotnik and McGuire, 1998; Zlotnik and Zurbuchen, 2002). The highly permeable aquifer of thickness $b=13 \mathrm{~m}$ consists of unconsolidated sands and gravels. Previous studies suggest that procedures to minimize the formation of well skin during installation were effective (Zurbuchen et al., 2002).

A mechanical flange-type packer consisting of two $1-\mathrm{cm}$ thick rubber disks spaced $10 \mathrm{~cm}$ apart was fitted on the EBF to prevent by-pass flow within the well. The profiles of flow rate through the EBF collected sequentially from the base of the well to near the top of the well screen at vertical increments of $15 \mathrm{~cm}$ during ambient flow conditions indicated no detectable ambient flow. Flow rate profiles under nonpumping conditions detected no ambient flow. Flow rate profiles under pumping conditions were collected in the same manner after allowing drawdown in the well to stabilize. The pumping rate was $Q_{\mathrm{p}}=60 \mathrm{~m}^{3} /$ day. Repeat cumulative discharge profiles collected were practically identical. Due to constraints of the pump configuration and operation, profiles were collected below depth $2 \mathrm{~m}$ from the water table to the aquifer base.

The EBF is capable of providing estimates of relative $K_{r, i}$ only in highly permeable aquifers. However, corrections of the EBF interpretation require the absolute estimates of $K_{r, i}$ in Eq. (45). In our case, we derive the averaged $K$ estimates from the available MLST for wells 14 and 15 over the appropriate part of the screen. The head losses function is taken from Eq. (18) with $C_{\mathrm{d}}=0.90$ (Arnold, 1997). Applying Eq. (1) directly to the field data yields $\left(K_{i} / K\right)_{\text {non-ideal }}$ estimates. The appropriate $K$ profile estimates are shown in Fig. 5 for both wells. The MLST results are also displayed and are considered to be baseline values of $K_{i}$. Also, corrected for head losses $K$ estimates $\left(K_{i} / K\right)_{\text {ideal }}$ are also shown in the same figure.

The MLST and BFT data indicate that $K_{i}$ is highly variable with depth, ranging over an order of magnitude. In both wells there is an apparent increase in $K_{i}$ with depth that is common in alluvial aquifers. In well 14 there is a sharp increase in $K_{i}$ at the depth $10.5 \mathrm{~m}$ below top of casing, and a similar increase is seen in well 15 at depth $8.5 \mathrm{~m}$.

Corrected for head losses $\left(K_{i} / K\right)_{\text {ideal }}$ data from BFT are significantly greater than the $\left(K_{i} / K\right)_{\text {non-deal }}$ over practically the entire well, with the exception of aquifer base. This is to be expected because consideration of head losses assumes that more discharge enters the well section above the EBF compared to the case of ideal EBF at the same elevation. Additionally, since we were unable to collect the flow rate profile for the uppermost section of well screen, the zone where the conventional interpretation overpredicts $K_{i}$, is absent.

Both interpretations of the BFT do not match the MLST data well. The overall trend of increasing $K$ with depth in Fig. 5 is not that apparent if one applies the standard data interpretation (assumption of ideal EBF without head losses), albeit it matches the MLST local variations adequately. Effort to improve the overall trend using the correction for non-ideality of the EBF in Eq. (46) exaggerates existing discrepancies.

The idea of correcting the BFT data essentially exploits the aquifer homogeneity. For the MLST, 


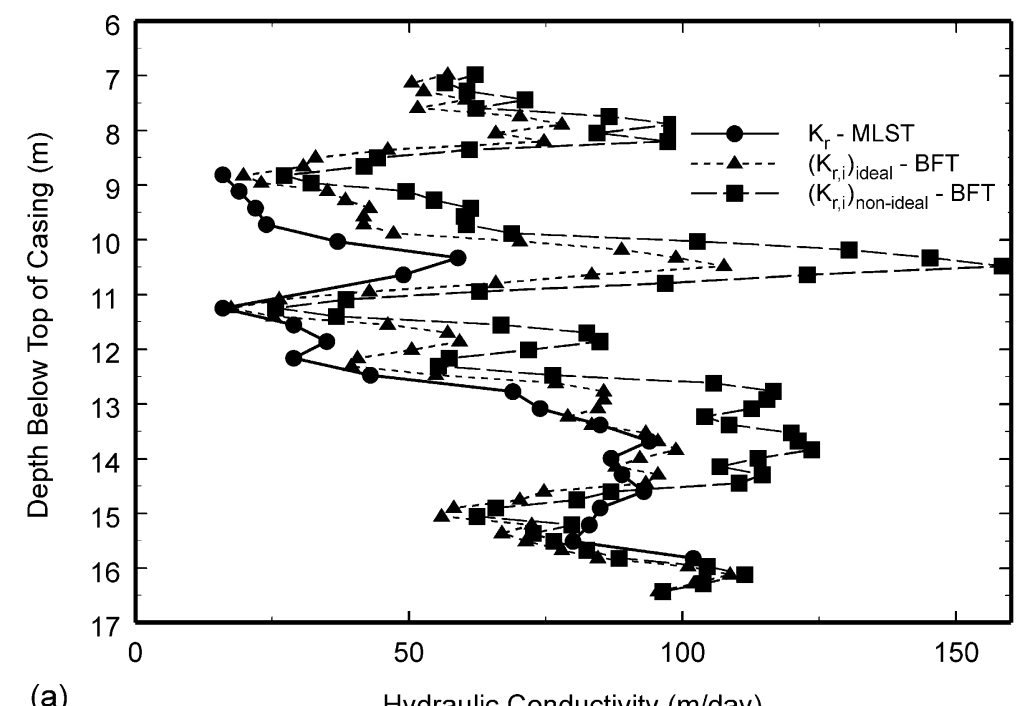

(a)

Hydraulic Conductivity (m/day)

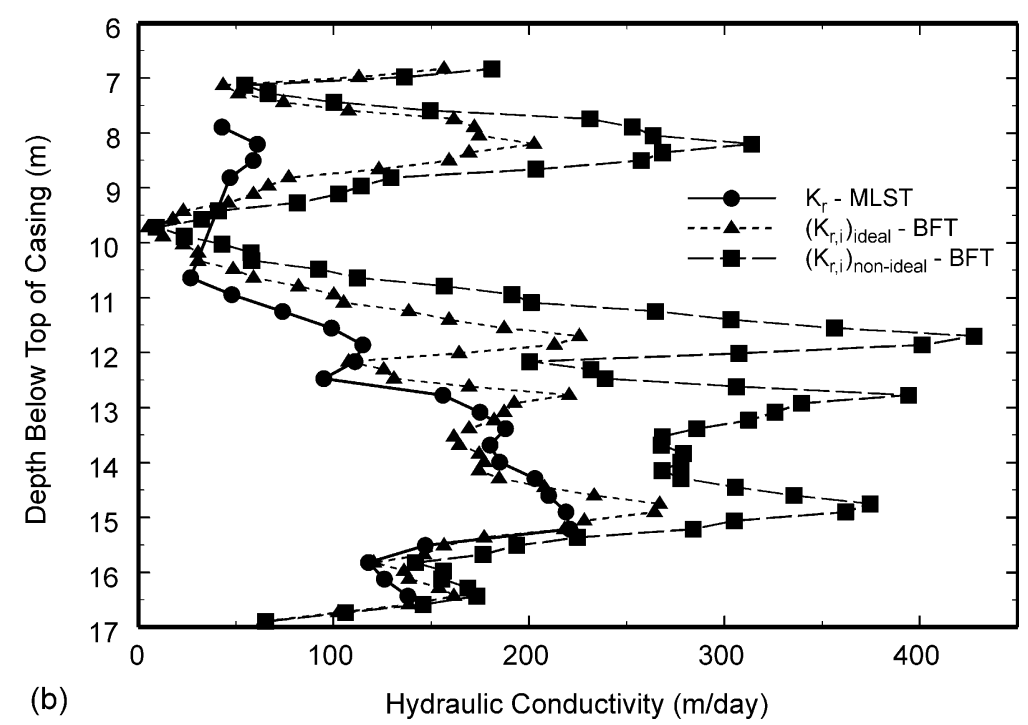

Fig. 5. Effect of the analytical correction for non-ideality of the EBF on estimates of hydraulic conductivity profiles: (a) well 14; (b) well 15 . (For comparison, the hydraulic conductivity profiles from the multi-level slug tests are also shown).

the assumption of local homogeneity is germane, because $K$ estimates are heavily biased towards the active screen zone (Zlotnik and Zurbuchen, 2003). However, during the BFT, flow is strongly dependent on the flow kinematics over the entire screen, and the resulting constant shape function is valid only for uniform aquifers. In heterogeneous aquifers, use of constant shape factor may not yield any improvements in the EBF test interpretations.

\section{Conclusions}

An analytical solution for the non-linear flow problem of the BFT has been obtained. This steadystate solution for drawdown and streamlines in the aquifer accounts for head losses across the EBF. This model represents the solution of the BFT problem as a superposition of the flow to a fully penetrating well and a dipole flow. Both drawdown and the Stokes' 
stream function are presented by infinite series. The model significantly reduces computational efforts for simulating flow in a homogeneous aquifer.

Application of the model was compared with the results of finite-difference models of Dinwiddie et al. (1999) and Ruud et al. (1999). This comparison indicates quantitative and qualitative correspondence with the results of numerical modeling. When head loss occurs across the EBF, flow in the aquifer is no longer horizontal and a larger proportion of flux to the well is distributed to the zone above the EBF than predicted assuming an ideal flowmeter without head losses. The amount of by-pass flow is dependent on the functional head loss characteristics of the EBF, $K_{r}$ of the aquifer, elevation of the EBF in the well, aquifer thickness, well radius, and anisotropy.

The derived analytical solutions for cumulative and differential discharge as a function of EBF elevation also confirmed the findings of Ruud et al. (1999) that apparent $K_{r}$ for the zone below the EBF is decreased and $K_{r}$ is increased for the zone above the EBF. The solution also supports the findings of Dinwiddie et al. (1999) on the decrease of apparent $K$ in the lower $85 \%$ section of the well and increase of apparent $K$ in the upper $15 \%$ of the well section length.

Albeit effective for uniform aquifers, this solution has a limited potential for applications in heterogeneous conditions. Comparison with independently obtained $K$ profiles by MLST indicates that corrections for head losses in EBF data cannot be easily done by modification of data interpretation only, and more sophisticated field procedures are needed.

The obtained solution provides additional insights into the kinematics of the BFT and can provide useful guidance for proper applications of the EBF and BFT.

\section{Acknowledgements}

This work was supported by the USGS grant HQ96GR02683, Water Center, and Department of Geosciences, University of Nebraska-Lincoln (UNL). We are grateful to Fred Molz, Clemson University, for discussions and information on hydraulic characteristics of the EBF, Carl McElwee, University of Kansas and an anonymous reviewer for numerous suggestions that led to improvements of the manuscript.

\section{References}

Arnold, K.B., 1997. Calibration of an electromagnetic borehole flowmeter in an artificial well without a packer. Masters thesis, Clemson University.

Bear, J., 1972. Dynamics of Fluids in Porous Media, Elsevier, New York, 764 pp.

Boman, G.K., Molz, F.J., Boone, K.D., 1997. Borehole flowmeter application in fluvial sediments: methodology, results, and assessment. Ground Water 35 (3), 443-450.

Cole, K., Zlotnik, V., 1994. Modification of Dagan's numerical method for slug and packer test interpretation. In: Peters, A., et al. (Eds.), Computational Methods in Water Resources X, vol. 1. Kluwer Academic Publishers, Dordrecht, pp. 719-726.

Dinwiddie, L.C., Foley, N.A., Molz, F.J., 1999. In-well hydraulics of the electromagnetic borehole flowmeter. Ground Water 37 (2), 305-315.

Foley, N.A., 1997. Pressure distribution around an electromagnetic borehole flowmeter in an artificial well. Masters thesis, Clemson University.

Fox, R.W., McDonald, A.T., 1992. Introduction to Fluid Mechanics, Wiley, New York, 829 pp.

Hamming, R.W., 1962. Numerical Methods for Scientists and Engineers, McGraw-Hill, New York.

Hess, A.E., 1986. Identifying hydraulic conductive fractures with a slow-velocity borehole flowmeter. Can. Geotech. J. 23, 69-78.

Hufschmied, P., 1986. Estimation of three-dimensional statistically anisotropic hydraulic conductivity field by means of single well pumping test combined with flowmeter measurements. Hydrogeologie 2, 163-174.

Javandel, I., Witherspoon, P.A., 1969. A method of analyzing transient fluid flow in multilayered aquifers. Water Resour. Res. 5, 856-869.

Molz, F.J., Young, S.C., 1993. Development and application of borehole flowmeters for environmental assessment. Log Anal. 3, 13-23.Jan.-Feb..

Molz, F.J., Morin, R.H., Hess, A.E., Melville, J.G., Güven, O., 1989. The impeller meter for measuring aquifer permeability variations: evaluations and comparisons with other tests. Water Resour. Res. 25 (7), 1677-1686.

Paillet, F.L., Hess, A.E., Cheng, C.H., Hardin, E.L., 1987. Characterization of fracture permeability with high-resolution vertical flow measurements during borehole pumping. Ground Water 25 (1), 28-40

Rehfeldt, K.R., Hufschmied, P., Gelhar, L.W., Schaefer, M.E., 1989. The borehole flowmeter technique for measuring hydraulic conductivity variability. Report No. EN 6511, Electric Power Research Institute, Palo Alto, CA.

Ruud, N.C., Kabala, Z.J., 1997. Response of a partially penetrating well in a heterogeneous aquifer: integrated well-face flux vs. uniform well-face flux boundary conditions. J. Hydrol. 194, 76-94.

Ruud, N.C., Kabala, Z.J., Molz, F.J., 1999. Evaluation of flowmeter-head loss effects in the flowmeter test. J. Hydrol. 224, 55-63.

Young, S.C., 1995. Characterization of high- $K$ pathways by borehole flowmeter and tracer tests. Ground Water 33 (2), 311-318. 
Young, S.C., 1998. Impacts of positive skin effects on borehole flowmeter tests in a heterogeneous granular aquifer. Ground Water 36 (1), 67-75.

Young, S.C., Pearson, H.S., 1995. The electromagnetic borehole flowmeter: description and application. Ground Water Monitor. Rev. 15 (4), 138-147.

Zlotnik, V., Ledder, G., 1994. Effect of boundary conditions on dipole flow. In: Peters, A., et al. (Eds.), Computational Methods in Water Resources X, vol. 2. Kluwer Academic Publishers, Dordrecht, pp. 907-914.

Zlotnik, V., Ledder, G., 1996. Theory of dipole flow in uniform anisotropic aquifers. Water Resour. Res. 32 (3), 1119-1128.
Zlotnik, V.A., McGuire, V.L., 1998. Multi-level slug tests in highly permeable formations. 2. Hydraulic conductivity identification, method verification, and field applications. J. Hydrol. 204, 283-296.

Zlotnik, V.A., Zurbuchen, B.R., 2003. Field study of hydraulic conductivity in a heterogeneous aquifer: comparison of single-borehole measurements using different instruments. Water Resour. Res., 39 (4), 1101, doi: 10.1029/ 2002 WR001415.

Zurbuchen, B.R., Zlotnik, V.A., Butler, J.J. Jr., 2002. Dynamic interpretation of slug tests in highly permeable aquifers. Water Resour. Res. 38 (3), doi: 10.1029/2000WR000197. 